\title{
FRACTAL MODEL FOR SKIN CANCER DIAGNOSIS USING PROBABILISTIC CLASSIFIERS
}

\author{
Stalin Jacob, \\ Faculty of Engineering and Technology, \\ Botho University, \\ Botswana, Africa. \\ stalin.jacob@bothouniversity.ac.bw \\ Jenifer Darling Rosita, \\ Department of Electrical Engineering, \\ New era College, \\ Botswana, Africa. \\ jrosita@neweracollege.ac.bw
}

Submitted: Jan, 15, 2021 Revised: Apr, 08, 2021 Accepted: Apr, 23, 2021

\begin{abstract}
The early detection of skin cancer can lead to high prognosis rate. Thus it is very important to identify abnormalities in skin as early as possible. However, the detection of abnormalities at their early stages is a challenging task since the shape and colour of the abnormalities vary with different persons. In this study, fractal model for skin cancer diagnosis is developed. Differential Box Counting (DBC) method is implemented to get the fractal dimension from the dermoscopic images from two databases; International Skin Imaging Collaboration (ISIC) and $\mathrm{PH}^{2}$ database. The fractal features are classified using a parametric and non-parametric classification approach. The system provides promising results for skin cancer diagnosis with $96.5 \%$ accuracy on $\mathrm{PH}^{2}$ images and $91.5 \%$ accuracy on ISIC database images using the non-parametric classifier whereas parametric classifier gives $95 \%\left(\mathrm{PH}^{2}\right)$ and 90\% (ISIC) images.
\end{abstract}

Keywords: Skin cancer diagnosis, fractal model, parametric classification, non-parametric classification.

\section{INTRODUCTION}

Human skin comprises the largest share of weight of the human body. As the key organ guarding our internal tissues from outside intrusions, it is not rare tat skin itself is suffering from demagogical problems. For example, lesions are quite common on human skin. Though most lesions are risk free, skin cancer, a demagogical disease appearing like benign lesion occasionally might cause health problems. As melanoma incidence is increasing, many computerized diagnosis tools are developed and makes the diagnosis at the earliest.

The state-of-the-art research of skin lesions classification for the identification of cancer is systematically reviewed [1]. The dermoscopic image patterns are obtained from many public databases that employ the Convolutional Neural Network (CNN). Automated classification of skin lesions using Deep Convolutional Neural Networks (DCNN) for deep feature extraction from the finegrained variable images [2]. An Artificial Intelligence (AI) with DNN is demonstrated, and the mobile devices for classifying the skin cancer to the dermatologist as an easier and low-cost classification level. 
The dermoscopic classification method of skin cancer utilizes Support Vector Machine (SVM) and DCNN [3]. The RGB dermoscopic skin images are obtained from the open databases. These images are cropped using the filtering method to remove noises, including the hair and background of the pixel images. The pre-trained AlexNet CNN model is utilized in this article to extract feature characteristics and the SVM classifier for the skin cancer classification.

Combining human and AI with the gradient boosting method of approach assists the superior skin cancer classification [4]. The primary end-point features are used to categorize the skin images based on five classes. The secondary endpoint aids the binary classification of either benign or malignant skin tumors. Melanoma and Non-melanoma classification of skin cancer employ the k-means clustering model for the color and textural features extraction [5]. In this, the decomposition of sub-band levels of the skin images and the reconstruction of images provide six color-texture features that are fed to the classifier model for accurate classification.

Faster Region-based CNN (FRCNN) model is trained using the pigmented skin lesions dataset extraction of malignant melanoma tumors and the malignant basal cell carcinoma that differs from the benign tumors [6]. This CNN model is developed with a deep learning accuracy classification system for skin dermoscopic images. When diagnosed and screened at an early stage, melanoma patients can control the death rate due to skin cancer [7]. Excessive exposure to ultraviolet rays is the major cause. The inability to accurately classify skin cancer by dermatologists leads to developing the automated, efficient melanoma and nonmelanoma classification system employing transfer learning for the diagnosis and proper treatment.

Automatic Lesion Detection System (ALDS) [8], the skin cancer classification framework developed that initially utilizes watershed masking and active contours with a probabilistic approach to extract selected features analysis. Then SVM and the Neural Classifier model are used for the classification of the dermoscopic images. A comparative study of melanoma cancer type features such as color, texture, and shapes are extracted by employing the linear normalization of pre-processing as the feature selection input image dataset [9]. Then the Balanced Random Forest (RF) classifier among six different classifiers provides a better classification system for skin cancer. Melanoma skin cancer is very dangerous, and if we treat them using ultraviolet radiation, then the skin cell DNA is also damaged [10]. Hence, the CAD-based deep learning method of CNN for extracting the feature image datasets and the LeNet-5 architecture assists the dermoscopic tumor image classification.

The input RGB skin images obtained from the databases are initially converted into gray level resized luminant pixel elements suitable for feature extraction utilizing the Otsu method of feature localization [11]. Then the fusion of extracted deep features from the pre-trained Alex net and VGG16 are integrated using wavelet transform with CNN. Then, the Principle Component Analysis (PCA) classification method into the benign or malignant tumor is performed. AI-based digital image classification has more advancements than dermatologists and clinicians in diagnosing skin cancers [12]. This enhances the future opportunities in improving the automated early diagnosis of different skin cancer types and its appropriate treatment with higher accuracy than dermatologists.

\section{METHODS AND MATERIALS}

The overall flow of skin cancer diagnosis using dermoscopic images is shown in Figure 1. In this study, the dominant features are extracted from the 
fractal model which is implemented using DBC method. The fractal dimensions from the fractal modeled skin images are classified into benign or malignant using parametric and non-parametric classifiers.

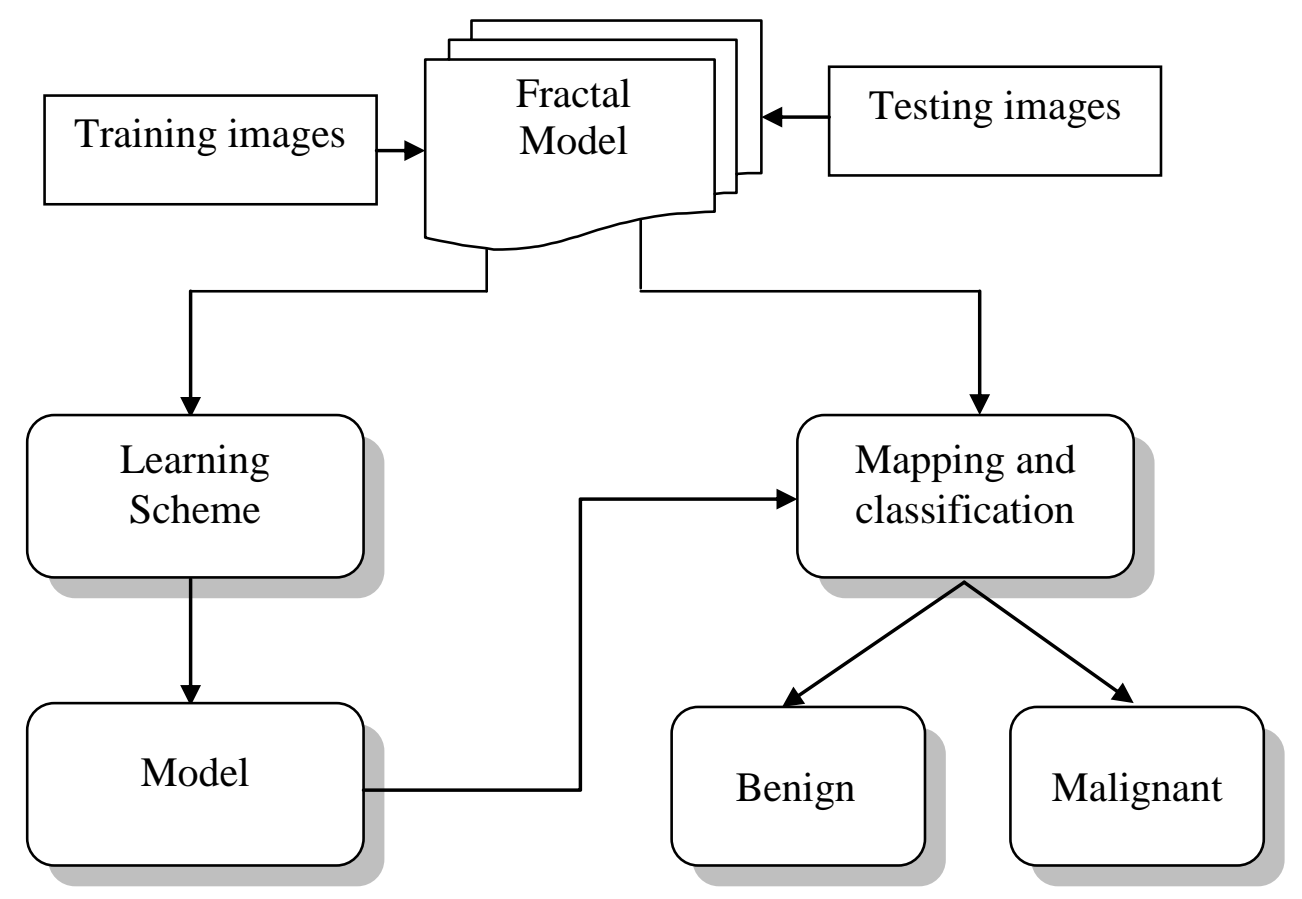

Fig. 1 Overview of skin cancer diagnosis system

\section{A. Fractal Model for Feature Extraction}

There is no universally accepted definition of a fractal, however the following tentative definition is offered in [13]." A fractal is by definition a set for which the Hausdorff-Besicovitch dimension strictly exceeds the topological dimension". The topological dimension is the ordinary Euclidean dimension of the space in which the set exists. The fractal model has been proposed as a model for natural textures [14], the key parameter of the model being the fractal dimension. The previous 'definition' of a fractal and its dimension cannot be used directly in the estimation, as the $d$-measure cannot be obtained as $\delta$ tends to zero. However, a variety of alternative methods have been used in an attempt to measure the fractal dimension of textures such as box counting, Fourier spectrum analysis, blanket method and spatial correlation analysis.

Fractal descriptor, a scalar called the Fractal Dimension (FD) which is defined as a ratio of the number of features at one scale to the number of features at the next scale. It is a number that quantifies the degree of surface roughness by examining similar structures on an image at different scales (elements being replica of the original structure) hence it is a self-similarity measure. The FD is a mixed space-scale representation with the scaling embedded in the spatial domain methods, for example, the box for estimating the FD is different at different resolution levels. Thus the dimension of a fractal surface contains information about the surface's geometrical properties. For textured images PD near 2 and 3 means a fine and coarse/rough texture respectively [99].

The FD is scale invariant and also invariant to linear transformations of the data and these attributes are keys in the implementation of a vision system. A 
surface is said to be fractal if the FD is stable over a wide range of scales. The local FD on a window adapts to variations in texture patterns on an image surface.

The implementation of the DBC [15] involves partitioning an image into boxes of size $s$. The $s$ is altered progressively to smaller sizes and each time counting $N$ the number of boxes covering the surfaces for each size of the box. A least squares fit is then performed using a plot of a graph of $\log N$ against $\log (1 / r)$ where $r=s / M$ to estimate FD which is shown in Eqn. (1).

$$
F D=\frac{\log \left(N_{r}\right)}{\log (1 / r)}
$$

where $N_{r}=\sum_{i, j} n_{r}(i, j)$. The above Eqn. (1) can be expressed in the form $y=m x+c$ which is $\log N_{r}=F D \log (1 / r)+C$. The pixel coordinates in the image are $i$ and $j$. Also,

$$
n_{r(i, j)=l-k+1}
$$

The differential part is illustrated in Eqn. (2). $k$ and $l$ boxes receives the minimum and maximum grey level in the $(i, j)^{\text {th }}$ grid respectively. The FD is obtained as a slope of this curve. The first order statistics (FOS) value within a given $s \times s$ box can be used to extract texture information. The features within a box hold the information contained in the texture.

The DBC has a larger dynamic range, it is faster to compute, it is more accurate and it is effective where the surface structure is self-similar. For many low-resolution images, the Box Counting $(\mathrm{BC})$ and $\mathrm{fBm}$ estimators are unreliable and generate inconsistent results. In $\mathrm{BC}$ the range of scale-invariance is assumed to be set by the physical size of the pixel yet the pixel size and the true range are unrelated.

\section{B. Parametric and Non-parametric Classification}

Most statistical classifiers use training data to directly or indirectly compare the probabilities of an input being from each class. In their purest form they estimate the class conditional probability density functions of the input, $\hat{p}(X \mid \omega)$, where $X$ represents the input as a random vector and $\omega$ represents a class.

The calculation of $\hat{p}(Y \mid \omega)$ for an input $Y$ and for each class $\omega$, the minimum error approach to classification requires that $Y$ should be assigned to the class, $\omega$, which maximizes $p(\omega \mid X=Y)$. Use of Baye's rule and noting that $p(X=Y)$ is unknown but equal for each case, leads to the conclusion that classification error is minimized by assigning $Y$ to the class which maximizes $\hat{p}(X=Y \mid \omega) \hat{p}(\omega)$.

The alternative is to use the maximum likelihood approach to classification, which requires that $Y$ should be assigned to the class, $\omega$, which maximizes $\hat{p}(X=Y \mid \omega)$. The difference between the two approaches is that the former minimizes the error by biasing the classification towards the more likely occurring classes.

Parametric and non-parametric classifiers differ in their approaches to the estimation of $\hat{p}(X \mid \omega)$. Parametric classifiers assume that the distributions have a 
particular form and estimate the parameters of the model from the training data. Non-parametric classifiers place no restrictions on the form of $\hat{p}(X \mid \omega)$ and provide a methodology for the estimation of the population distribution from a sample.

\section{B.1 Parametric Classification}

The quadratic classifier is a parametric classifier which assumes that samples from each class are from multivariate normal distributions with different mean vectors and covariance matrices. This an appropriate model in many real applications due to the central limit theorem. The expression used to estimate $\hat{p}(X=Y \mid \omega)$ where $Y$ is a particular instance of the random variable $X$ is shown in Eqn. (3),

$$
\hat{p}(X \mid \omega=m)=\frac{1}{(2 \pi)^{n / 2}\left|\sum_{m}\right|^{1 / 2}} \exp \left(-\frac{1}{2}\left(X-M_{m}\right) \Sigma_{m}^{-1}\left(X-M_{m}\right)\right)
$$

where $n$ represents the dimensionality of the event space, $\Sigma_{m}$ is the covariance matrix of class $m$ and $M_{m}$, is the mean vector. In practice $\hat{p}(X=Y \mid \omega)$ is usually not calculated as the classification can be reduced to comparison of quadratic distances.

\section{B.2 Non-parametric Classification}

The Parzen classifier is a non-parametric classifier which estimates $\hat{p}(X=Y \mid \omega)$ where $Y$ is a particular instance of the random variable $X$, by calculating a sum over all the training values in $\omega$ which is weighted according to a normalized distance measure. The expressions used are shown in Eqns. (4) and (5).

$$
\begin{gathered}
\hat{p}(X \mid \omega=m)=\frac{1}{N} \sum_{i=1}^{N} k\left(X, X_{(i, m)}\right) \\
k\left(X, X_{(i, m)}\right)=\frac{1}{\sqrt{\left(2 \pi r^{3}\right)^{n}\left|\sum_{m}\right|}} \exp \left(-\frac{1}{2 r^{2}}\left(X-X_{(i, m)}\right) \sum_{m}^{-1}\left(X-X_{(i, m)}\right)\right)
\end{gathered}
$$

where $n$ represents the dimensionality of the event space, $\Sigma_{m}$ represents the covariance matrix of class $m$ which is estimated from the training data, and $r$ is the Parzen window kernel size which determines the range of influence of training samples. Determination of an appropriate value for $r$ is performed experimentally using the training data. Note that the distance measure used is the quadratic distance scaled by the covariance matrix; this ensures that the event space is scaled appropriately so as to prevent the over emphasis of highly correlated dimensions and dimensions with a large range (and vice versa). 


\section{RESULTS AND DISCUSSION}

Two databases; $\mathrm{PH}^{2}[16$ ] and ISIC [17] database are used for performance evaluation. $\mathrm{PH}^{2}$ database consists of 200 images (120 abnormal and 80 normal) where as ISIC has 2000 images (1372 malignant and 628 benign ). To analyze the effectiveness of the system, sensitivity and specificity are important quantitative measures. In this study, sensitivity means the success rate of diagnosing true malignant lesion as malignant while specificity means the success rate of diagnosing true benign lesion as benign. Figure 2 and 3 show the performances of parametric and non-parametric classification on $\mathrm{PH}^{2}$ database images and ISIC database images respectively.

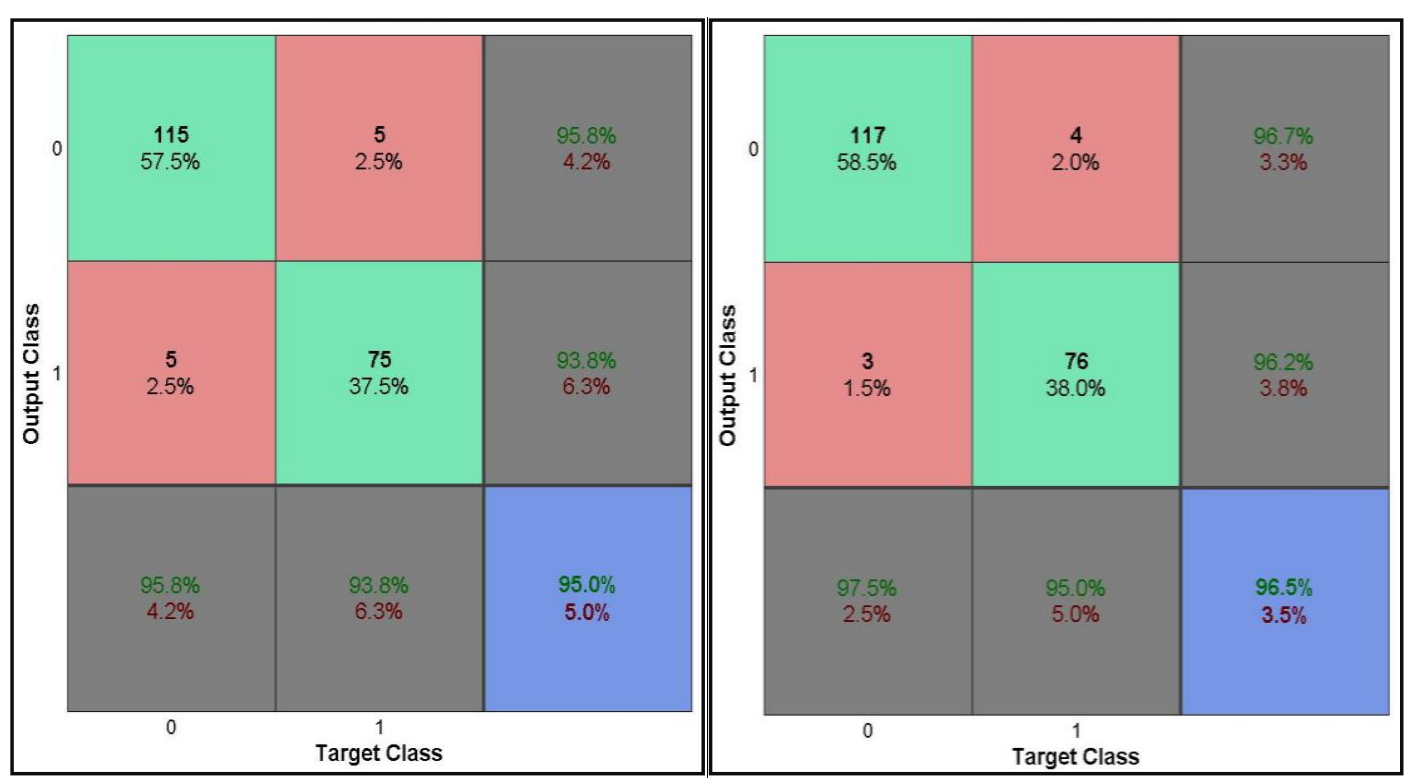

(a)

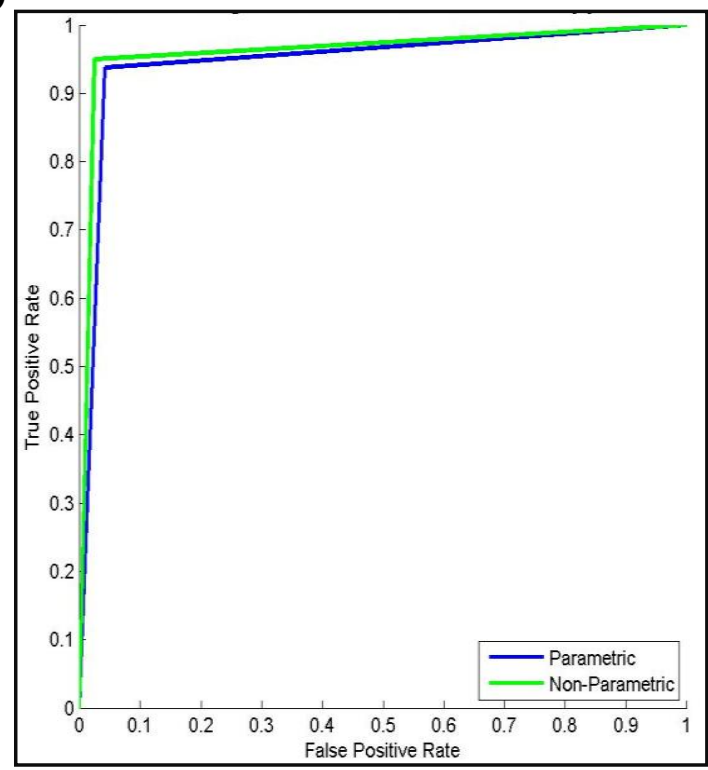

(c)

Fig. 2 Performance on PH2 database images (a) Parametric classification (b) Non-parametric classification (c) combined ROCs 
Int. J.Adv.Sig.Img.Sci, Vol.7, No.1, 2021
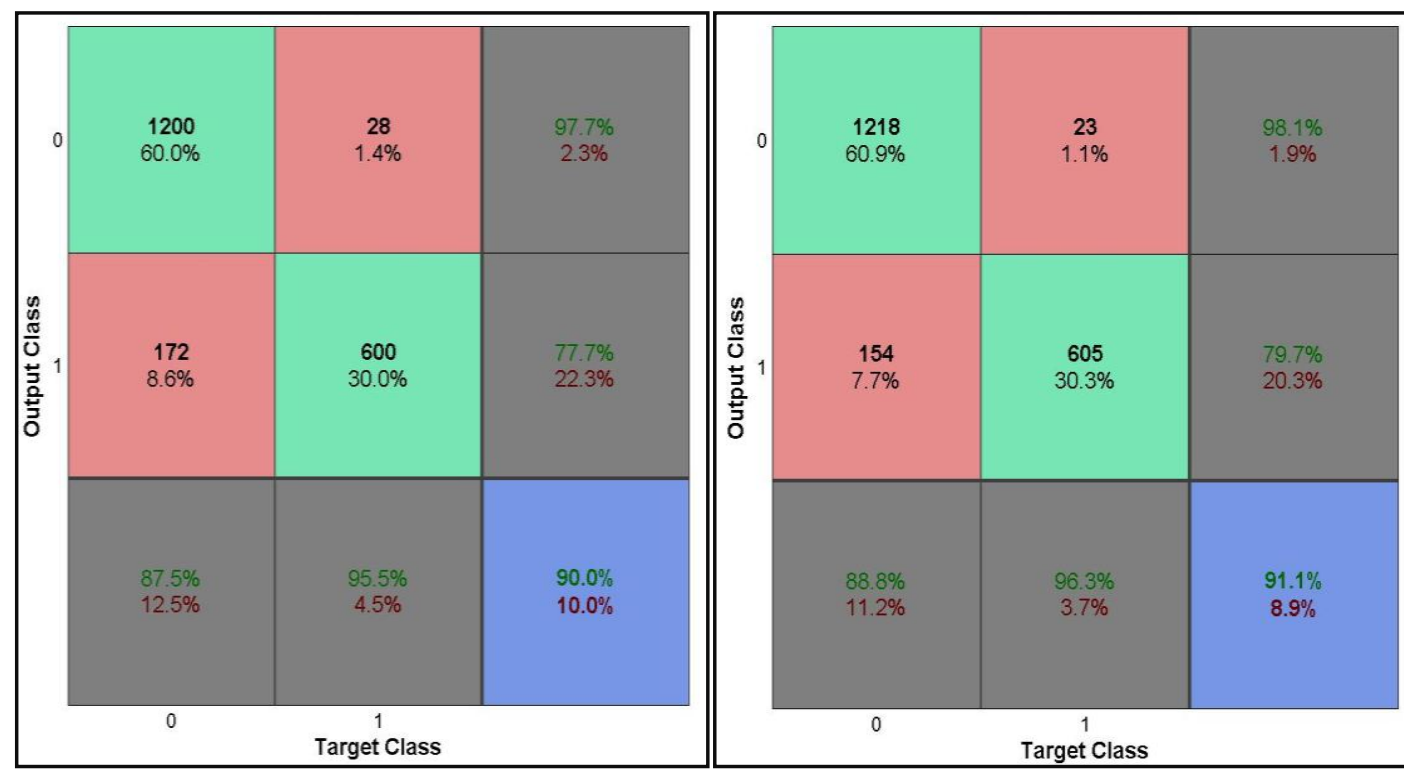

(a)

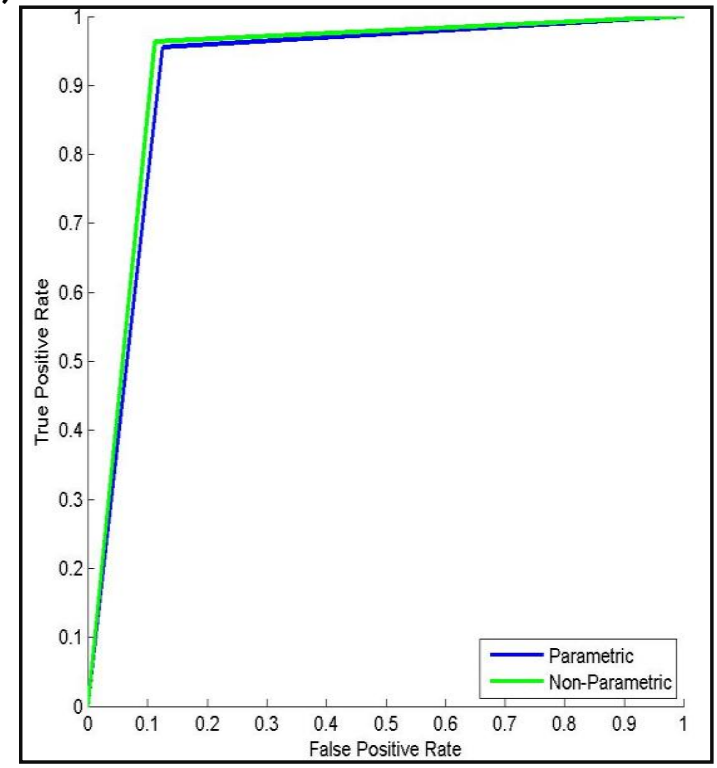

(c)

Fig. 3 Performance on ISIC database images (a) Parametric classification (b) Non-parametric classification (c) combined ROCs

It is inferred that non-parametric classification gives better results than parametric classification on $\mathrm{PH}^{2}$ and ISIC database images. The classification accuracy of non parametric classifier is $96.5 \%$ and $91.5 \%$ for $\mathrm{PH}^{2}$ and ISIC database. The sensitivity which is the main parameter that gives the correct classification of abnormality is $97.5 \%$ and 88.8 by non-parametric classification whereas it is $95.8 \%$ and 87.5 by parametric classification for $\mathrm{PH}^{2}$ and ISIC database. It is also observed that the specificity of the system is also higher for non-parametric classification. 


\section{CONCLUSION}

In this paper, fractal model is presented for skin cancer diagnosis using dermoscopic images. The proposed computerized system for skin cancer diagnosis has several advantages. Firstly, it is a non invasive procedure and secondly it gives a detailed record of lesion. The computed fractal dimension by DBC method is used for predicting the abnormalities in skin images. The prediction is made using a parametric classifier and a non-parametric classifier. The evaluation of the proposed system uses 10-fold cross-validation on $\mathrm{PH}^{2}$ and ISIC images. Results show that the non-parametric classification is better than parametric classification. In future, the performance of the system can be increased by including an effective preprocessing step to remove hairs and noises in the skin images.

\section{REFERENCES}

[1]. T.J. Brinker, A. Hekler, J.S. Utikal, N. Grabe, D. Schadendorf, J. Klode and C. Von Kalle, "Skin cancer classification using convolutional neural networks: systematic review", Journal of medical Internet research, Vol. 20,No. 10, 2018, pp. e11936

[2]. A. Esteva, B. Kuprel, R.A. Novoa, J. Ko, S.M. Swetter, H.M. Blau and S. Thrun, "Dermatologist-level classification of skin cancer with deep neural networks", Nature, Vol. 542, No. 7639, 2017, pp. 115-118.

[3]. U.O. Dorj, K.K. Lee, J.Y. Choi, and M. Lee, "The skin cancer classification using deep convolutional neural network", Multimedia Tools and Applications, Vol. 77, No. 8, 2018, pp. 9909-9924.

[4]. A. Hekler, J.S. Utikal, A.H. Enk, A. Hauschild, M. Weichenthal, R.C. Maron and A. Thiem, "Superior skin cancer classification by the combination of human and artificial intelligence", European Journal of Cancer, Vol. 120, 2019, pp. 114-121.

[5]. M. Anas, K. Gupta, and S. Ahmad, "Skin cancer classification using Kmeans clustering", International Journal of Technical Research and Applications, Vol. 5, No.1, 2017, pp. 62-65.

[6]. S. Jinnai, N. Yamazaki, Y. Hirano, Y. Sugawara, Y. Ohe and R. Hamamoto, "The development of a skin cancer classification system for pigmented skin lesions using deep learning”, Biomolecules, Vol. 10, No. 8, 2020, pp. 1123.

[7]. S.S. Chaturvedi, K. Gupta, and P.S. Prasad, "Skin lesion analyser: an efficient seven-way multi-class skin cancer classification using MobileNet", In International Conference on Advanced Machine Learning Technologies and Applications, 2020, pp. 165-176.

[8]. M.A. Farooq, M.A.M. Azharand and R.H. Raza, "Automatic lesion detection system (ALDS) for skin cancer classification using SVM and neural classifiers", IEEE 16th International Conference on Bioinformatics and Bioengineering, 2016, pp. 301-308.

[9]. T.C. Pham, G.S. Tran, T.P. Nghiem, A. Doucet, C.M. Luong and V.D. Hoang, "A comparative study for classification of skin cancer", International Conference on System Science and Engineering, 2019, pp. 267-272.

[10]. R. Refianti, A.B. Mutiara and R.P. Priyandini, "Classification of melanoma skin cancer using convolutional neural network", IJACSA, Vol. 10, No. 3, 2019, pp. 409-417. 
[11]. J. Amin, A. Sharif, N. Gul, M.A. Anjum, M.W. Nisar, F. Azam and S.A.C. Bukhari, "Integrated design of deep features fusion for localization and classification of skin cancer", Pattern Recognition Letters, Vol. 131, 2020, pp. 63-70.

[12]. M. Goyal, T. Knackstedt, S. Yan and S. Hassanpour, "Artificial intelligencebased image classification for diagnosis of skin cancer: Challenges and opportunities", Computers in Biology and Medicine, 2020, pp. 104065.

[13]. B. Mandelbrot. The Fractal Geometry of Nature. W. H. Freeman and Co., 1982.

[14]. A. Pentland, "Fractal-based description of natural scenes", IEEE Computer Society Conference on Computer Vision and Pattern Recognition, 1983, pp. 201-209.

[15]. N. Sarkar and B. Chaudhuri, "An efficient approach to estimate fractal dimension of textural images", Pattern Recognition letters, Vol. 25, 1992, pp. 1035-1041.

[16]. T. Mendonça, P.M. Ferreira, J.S. Marques, A.R. Marca and J. Rozeira, "PH2-A dermoscopic image database for research and benchmarking", 35th Annual International Conference on Engineering in Medicine and Biology Society, 2013, pp. 5437-5440.

[17]. N. Codella, D. Gutman, M.E. Celebi, B. Helba, M.A. Marchetti, S. Dusza, A. Kalloo, K. Liopyris, N. Mishra, H. Kittler and A. Halpern, "Skin Lesion Analysis Toward Melanoma Detection", A Challenge at the 2017 International Symposium on Biomedical Imaging (ISBI), Hosted by the International Skin Imaging Collaboration (ISIC)". arXiv: 1710.05006 [cs.CV] Available: https://arxiv.org/abs/1710.05006 